\title{
Ordered Mesoporous Silica Materials for Protein Adsorption
}

\author{
F. Sezões ${ }^{a}$, M.M.L. Ribeiro Carrott ${ }^{b}$, P.A.M. Mourão ${ }^{c}$, P.A. Russo ${ }^{d}$ \\ and P.J.M. Carrott ${ }^{\mathrm{e}}$
}

Centro de Química de Évora and Departamento de Química, Universidade de Évora, Escola de Ciências e Tecnologia, Colégio L. A. Verney, 7000-671 Évora, Portugal

afsezões@gmail.com, 'bmanrc@uevora.pt, 'pamm@uevora.pt, 'pafrusso@uevora.pt, peter@uevora.pt

Keywords: MCF; SBA-15; MCM-41; Lysozyme adsorption; Bovine serum albumin adsorption

\begin{abstract}
Lysozyme and BSA were used, as model proteins of considerably different dimensions, in order to evaluate the influence of the distinct pore structural characteristics of three types of ordered mesoporous silica materials (MCF, SBA-15 and MCM-41) on protein adsorption. Characterisation by $\mathrm{X}$ ray diffraction and nitrogen adsorption at $77 \mathrm{~K}$ revealed the typical pore structural features of each type of material. The maximum of the pore size distributions indicated that the width of the windows of MCF (2) (mesitylene/P123 of 2) was larger than the pore diameter of the unidirectional tubular pores of SBA-15. All the materials presented similar small external surface areas but high pore volumes, with that of $\mathrm{MCF}(2)$ being the highest. The adsorption of lysozyme at $\mathrm{pH}=8$ increased in the order MCM-41 $<<\mathrm{SBA}-15<\mathrm{MCF}(2)$, and the uptakes were well above those of $\mathrm{BSA}$ at $\mathrm{pH}=5$. Although BSA is not completely excluded from the mesopores of SBA-15 and MCF (2), as happens with MCM-41, the adsorption occurs to a very limited extent. The overall behaviour of these SBA-15 and MCF (2) samples was not significantly different and both revealed potential for the separation of these proteins.
\end{abstract}

\section{Introduction}

The unique structural properties of ordered mesoporous materials, disclosed in 1992 for the M41S family [1], stimulated an intensive research in many fields of materials science. Besides the high surface areas and pore volumes and the uniformity of the pore structure, the possibility of tuning the geometry and the pore size to a target dimension in the mesopore range are extraordinary features that lead to a great variety of new regular materials. In particular, those with wider mesopores are attractive for their use as host matrices for immobilisation, adsorption and encapsulation of biomolecules, having potential for application in many fields, such as in catalysis and separation, and as biosensors and drug delivery systems [2, 3, 4].

Several studies have shown that protein adsorption is sensitive to several factors, including the pore size and surface chemistry of the mesoporous materials, the dimensions and isoelectric point of the protein and the solution conditions [2]. The complexity of the protein adsorption process requires that studies in different materials are carried out under similar conditions, otherwise the comparison of the results obtained is not straightforward.

In this work, we focus our study on the adsorption from aqueous solution of two biomolecules with different size, namely, Bovine serum Albumin (BSA) which has large dimensions and Lysozyme which is a comparatively small enzyme, in ordered mesoporous silicas with different pore systems and sizes, namely, MCF, SBA-15 and MCM-41. MCFs (Mesostructured Cellular Foams) are a family of materials composed by large spherical cells that are interconnected by uniform windows, whose size can be tuned by controlling synthesis parameters. On the other hand, the materials belonging to SBA-15 and MCM-41 types both have unidirectional cylindrical mesopores, in a hexagonal array, with SBA-15 having wider mesopores interconnected by microporosity in the thicker silica walls. To our knowledge, the comparison of adsorption, under the same conditions, of lysozyme or BSA on these three types of materials has not been presented. 